\title{
Análisis bibliométrico de la producción científica en el fútbol sala
}

\author{
Bibliometric analysis of scientific production in futsal
}

\author{
Palazón, M.A., Ortega, E. y García-Angulo, A.
}

Facultad de Ciencias del Deporte. Universidad de Murcia. Campus de Excelencia Internacional Regional "Campus Mare Nostrum", Murcia, España

\begin{abstract}
Resumen: El objetivo del presente estudio es realizar un análisis bibliométrico de la producción científica en el deporte de fútbol sala. Para ello se utilizó la colección principal de la plataforma "Web of Science" con las bases de datos Science Citation Index Expanded (SCI-EXPANDED) y Social Sciences Citation Index (SSCI). La búsqueda ofreció 81 artículos de los que se analizaron las siguientes variables: 1) título; 2) nombre del autor principal; 3) institución del primer autor; 4) $\mathrm{n}^{\circ}$ de autores; 5) revista; 6) número de citas; 7) año de publicación; 8) disciplina principal del estudio; 9) tipo de estudio; 10) tamaño de muestra; 11) nivel deportivo de la muestra, 12) sexo de la muestra. En los resultados destacaron los artículos de tipo experimental, los de sexo masculino y a deportistas de nivel profesional. Respecto a las instituciones, destacan las de países con un gran auge en lo que se refiere a fútbol sala, como Brasil y Portugal.
\end{abstract}

Palabras Clave: futsal, fútbol sala, bibliometría, producción científica. Abstract: The aim of this study is to conduct an analysis of the scientific production in the sport of futsal. To do this the main collection platform "Web of Science" with databases Science Citation Index Expanded (SCIEXPANDED) and Social Sciences Citation Index (SSCI) was used. The search provided 81 articles of which the following variables were analyzed: 1) title; 2) name of the first author; 3) institution of the first author; 4) number of authors; 5) magazine; 6) number of citations; 7) year of publication; 8) main discipline of study; 9) type of study; 10) sample size; 11) sport level of the sample, 12) sex shows. The results highlighted the experimental items, the male and a professional level. Concerning institutions, highlights countries with booming in regards to football, as Brazil and Portugal.

Keywords: futsal, futsal, bibliometrics, scientific production.

\section{Introducción}

La cienciometría es la ciencia que estudia la producción científica con el fin de medirla y analizarla. En la práctica, la cienciometría suele ser llevada a cabo a través de la bibliometría, la medida de las publicaciones científicas. La bibliometría es una técnica que desarrolla sus unidades de medida basándose esencialmente, en la Bibliografía, las Fuentes de Información y en las Ciencias de la Documentación, base de todas las disciplinas señaladas (Carrizo, 2000).

La bibliometría es ante todo un corpus metodológico, inicialmente centrado en el análisis estructural y prognosis de la información, contenida principalmente en publicaciones de investigación científica (Fernández y Bueno, 1998). Es una parte de la cienciometría que aplica métodos matemáticos y estadísticos a toda la literatura de carácter científico y a los autores que la producen, con el objetivo de estudiar y analizar la actividad científica. Es una herramienta esencial ya que sobre ella se constituye la base sobre la cual se mide la producción científica (Carrizo, 2000).

El análisis de la actividad a través de indicadores bibliométricos permite profundizar en el estudio de la estructura y dinámica del proceso investigador, analizando en lo que es su mínima unidad de funcionamiento y complementando a otros enfoques, como los procedentes de la sociología de la ciencia. Pero además, estos estudios pueden aportar datos de

Dirección para correspondencia [Correspodence address]: María Ángeles Palazón. Facultad de Ciencias del Deporte. Universidad de Murcia. Campus de Excelencia Internacional Regional "Campus Mare Nostrum". Murcia (España).E-mail: mariaangeles.palazon@um.es interés para la política científica de un país (Zulueta, Cabrero y Bordons, 1999). El uso de indicadores bibliométricos ha ido en incremento como complemento de otros indicadores científicos para analizar la situación de la investigación de un país, su evolución en el tiempo y su posición en el contexto internacional (Zulueta et al., 1999).

En los últimos años se ha producido un crecimiento importante de la producción científica en las Ciencias de la Actividad Física y el Deporte (CCAFD), aparejado a la consolidación de su comunidad académica universitaria (Devís, Valenciano, Villamón y Pérez, 2010). Debido a este elevado incremento han empezado a aparecer estudios de carácter bibliométrico en los que se analiza tanto la calidad, como la cantidad de la investigación en las ciencias del Actividad Física y del Deporte. En este sentido se aprecian tres grandes líneas de investigación que aglutinan diferentes estudios de carácter bibliométrico. En concreto se trata de estudios que analizan las revistas científicas, estudios que analizan un área de investigación desde su perspectiva global a través de estudios de tesis, proyectos de investigación, congresos, etc., y finalmente un tercer gran grupo que analiza de manera pormenorizada la producción científica en especialidades deportivas concretas.

En el primer grupo se dedica al análisis de revistas científicas, ya sea para analizar su producción científica, comparándolas con otras revistas, el nivel de publicaciones, etc. Por ejemplo, Arbinaga, Aragón, y Tejedor, R. (2010), realizan un estudio bibliometrico de la Revista de Psicología del Deporte; Devís, Antolín, Villamón, Moreno y Valenciano (2003) revisan la calidad de las revistas; Devís, Villamón, Antolín, 
Valenciano y Moreno (2004) hablan de la adecuación a las normas ISO; Villamón, Devís, Valencia y Valenciano (2007) y Villamón, Devís y Valenciano (2005) comprueban la visibilidad que alcanza una publicación periódica mediante su difusión.

En el segundo gran grupo se realizan estudios en los que se analiza la producción científica de un área de conocimiento científico a través del estudio pormenorizado de los trabajos presentados a congresos, tesis doctorales, proyectos de investigación, etc. Olmedilla, Ortega, González y Villarejo (2013), realizan un análisis de las principales temáticas en los proyectos de investigación concedidos en el plan nacional y en el Consejo superior de deportes en el área de la psicología del deporte, mientras que Ortega, Valdivia, Villarejo y Olmedila (2014), lo hacen en el área de las ciencias del deporte. Por otro lado Pérez (2007) habla de las contribuciones a los congresos del área de Didáctica de la Expresión Corporal.

Y por último, en el tercer gran grupo, se analiza la producción científica de especialidades deportivas concretas, ya sea para la investigación de aspectos técnico-tácticos, aspectos fisiológicos, sociales, de medicina deportiva o salud, etc. (e.g. Balmaseda, 2014; Peset, Ferrer, Villamón, González, Toca, y Aleixandre, 2013; Marín, Ortín, Garcés de Los Fayos, y Tutte, 2013; Martínez, Moya, Garces de los Fayos, 2013; Martínez, Ruiz y Navarro, 2002; Villarejo, Palao y Ortega, 2010).

Esta última línea de investigación, se encuentra dividida a su vez en varios sub-grupos. En primer lugar, existen estudios de áreas genéricas como la Psicología del deporte, Medicina del deporte, Fisiología del deporte y estudios sobre deportes concretos. Olmedilla, Ortega, Abenza y Boladeras (2011) realizan una revisión de la producción científica en la relación de lesiones y psicología deportiva, Estrada (2013) analiza la producción científica respecto a la rehabilitación de lesiones, Fernández y Alvero (2006) hablan sobre la cineantropometría, y Rodríguez, Rodríguez, Torres y Urrego (2011) hacen una revisión de la producción científica en la bio-retroalimentación en el rendimiento deportivo.

En segundo lugar, se aprecia un escaso número de estudios bibliométricos que analizan una disciplina deportiva concreta (Balmaceda, 2014; Torrebadella 2012; Villarejo, Palao y Ortega, 2010). Sin embargo, las Ciencias de la Actividad Física y el Deporte tienen un carácter multidisciplinar y este tipo de estudios son necesarios. Por estas escasas publicaciones de estudios bibliométricos respecto a los deportes en concreto, y en particular al fútbol sala, el objetivo de este estudio es el de conocer las tendencias de investigación en el deporte del fútbol sala y el análisis de la producción científica respecto a este deporte.

\section{Método}

El estudio se realizó en la plataforma Web of Science, en la colección principal. Concretamente en las bases de datos
Science Citation Index Expanded (SCI-EXPANDED) y Social Sciences Citation Index (SSCI).

La muestra está formada por el total de artículos obtenidos tras una búsqueda en la base de datos Web of Science utilizando el topic "futsal or fútbol sala" $(\mathrm{n}=84)$, desde el ańo 2005 hasta el año 2014. Como criterio de inclusión, se definió que fuera un artículo que tratara el deporte del futbolsala. Por ello, de los 84 artículos encontrados, se analizaron 81 eliminado tres estudios al estar relacionados con robótica.

De la muestra seleccionada se analizaron las siguientes variables: 1) Título; 2) Nombre del autor principal; 3) Institución del primer autor. 4) No de autores; 5) Revista en la que fue publicado cada artículo; 6) Número total de veces que ha sido citado cada artículo; 7) Año de publicación; 8) Disciplina principal del estudio (se utilizó la propuesta de Devís et al. 2010); 9) Tipo de estudio según Hernández, Fernández y Baptista (2000) (experimentales, descriptivos, y/o correlacionales); 10) Tamaño de muestra; 11) Nivel deportivo de la muestra (profesional, amateur, de iniciación, o de varios niveles deportivos); 12) Sexo de la muestra.

\section{Resultados}

De los 81 artículos analizados, participan un total de 59 autores principales. El investigador Travassos fue el que mayor número de artículos sobre este deporte ha publicado $(\mathrm{n}=5)$. Le sigue Vilar con cuatro artículos. Correa, Serrano y Ayala coinciden con tres artículos cada uno sobre el fútbol sala. Y con dos artículos cada uno se encuentran los autores Milanez, De Moura, Moreira, Fonseca, Gomes, Dogramaci, Rebelo, Castagna y Tessitore.

En cuanto a las 52 instituciones que aparecen en los artículos estudiados, la que presenta mayor cantidad de artículos es la Universidad de Sao Paulo de Brasil, con seis artículos. Le sigue la Universidad Estadual Londrina, también de Brasil, y la Universidad Beira Interior de Portugal, con cinco artículos cada una. Cuatro artículos proceden de la Universidad Técnica de Lisboa, Portugal, y la Universidad Federal Minas Gerais, de Brasil. Con tres artículos se encuentra a la Universidad Evora de Portugal, la Universidad de Murcia, España, y la Universidad Roma Tor Vergata, de Italia. Y por último, con dos artículos se encuentra la Universidad Porto, de Portugal y la universidad Technol, Sch Leisure Sport y Tourism, de Australia.

Los años de publicación de los artículos en objeto de estudio se han comprendido entre 2005 y 2014. Predominan los artículos centrados en el fútbol sala en el año 2013, con 22 artículos, y los del año 2012 con 21 artículos. Sólo se publicó un artículo en el año 2005, y sólo dos en el 2007.

En la tabla 1, se aprecia el número de artículos y el porcentaje de las diferentes variables analizadas. 
Tabla 1. Número de artículos y porcentaje, según variable objeto de estudio.

\begin{tabular}{|c|c|c|}
\hline Variable & Categoría & Porcentaje de artículos \\
\hline \multirow{9}{*}{ Disciplina } & Biomecánica & $4,9 \%$ \\
\hline & Fisiología & $27,1 \%$ \\
\hline & Teoría entrenamiento & $24,6 \%$ \\
\hline & Actividad Física y Salud & $11,1 \%$ \\
\hline & Psicología & $9,8 \%$ \\
\hline & Medicina Deportiva & $6,1 \%$ \\
\hline & Nutrición & $2,4 \%$ \\
\hline & Didáctica & $3,7 \%$ \\
\hline & Sociología Deportiva & $2,4 \%$ \\
\hline \multirow{2}{*}{$\begin{array}{l}\text { Tipo de } \\
\text { estudio }\end{array}$} & Experimental & $65,4 \%$ \\
\hline & Descriptivo & $16,0 \%$ \\
\hline \multirow{3}{*}{$\begin{array}{l}\text { No Autores } \\
\text { por artículo }\end{array}$} & De 1 a 3 & $30,8 \%$ \\
\hline & De 4 a 6 & $60,4 \%$ \\
\hline & De 7 a 11 & $8,6 \%$ \\
\hline \multirow{5}{*}{ Citas } & No citados & $48,1 \%$ \\
\hline & De 1 a 5 & $32,1 \%$ \\
\hline & De 6 a 10 & $9,8 \%$ \\
\hline & De 11 a 20 & $4,9 \%$ \\
\hline & Más de 20 & $4,9 \%$ \\
\hline \multirow{3}{*}{$\begin{array}{l}\text { Tamaño de } \\
\text { la muestra }\end{array}$} & De 1 a 100 & $72,7 \%$ \\
\hline & De 101 a 300 & $20,4 \%$ \\
\hline & Más de 300 & $6,8 \%$ \\
\hline \multirow{4}{*}{$\begin{array}{l}\text { Nivel de la } \\
\text { muestra }\end{array}$} & Profesional & $75 \%$ \\
\hline & Amateur & $10 \%$ \\
\hline & Iniciación & $0 \%$ \\
\hline & Varios & $15 \%$ \\
\hline \multirow{3}{*}{$\begin{array}{l}\text { Sexo de la } \\
\text { muestra }\end{array}$} & Hombre & $75 \%$ \\
\hline & Mujer & $14,5 \%$ \\
\hline & Ambos & $10,4 \%$ \\
\hline
\end{tabular}

Al analizar las disciplinas de estudio de los artículos, en la tabla 1 se puede observar que hay un predominio importante de las disciplina médicas, destacando el área de la Fisiología, siguiéndole la Teoría del entrenamiento, y en menor medida de la Actividad física y salud.

Al analizar los tipos de estudios, los artículos analizados responden a dos tipos: los estudios experimentales, y en menor medida los estudios descriptivos.

De la tabla 1, se desprende que en los artículos objeto de estudio han participado un gran número de autores. Se han encontrado de 1 a 11 autores por artículo. Predominan los artículos con un número total de cinco autores, concretamente en 20 de los artículos. Le sigue de cerca con cuatro autores un total de 19 artículos. Los artículos con un gran número de autores son escasos. Sólo uno tiene 11 autores y dos se encuentran con 10. También existen pocos artículos con un solo autor o dos autores.

En la tabla 1 se observa, agrupando el número de autores por artículo, que hay un predominio de artículos de 4 a 6 autores, le sigue el grupo de pocos autores, y por último, la minoría se la llevan los artículos de 7 a 11 autores.

En cuanto a revistas en los que se publican artículos de esta temática, destaca la revista "Journal of Strength and Conditioning Research", con un total de 12 artículos. Le sigue la revista "Motriz-Revista de Educaçao Fisica" con un total de seis artículos. Con cinco artículos se aprecia la "Journal of Sports Science and Medicine" y la "Jornal of Sports Sciences". La "Revista Brasileira de Medicina do Esporte" ofrece cuatro de estos artículos.

En los artículos analizados, se ha encontrado que la gran mayoría, no han sido todavía citados en ISI-Thomposon. Predominan después, los artículos que han sido citados una vez. En la tabla 1 se pueden observar el total de número de citas de los artículos agrupados, y por lo tanto, comprobar que hay varios artículos que han sido citados un gran número de veces. Existen artículos que han sido citados más de 20 veces. El grupo predominante, después del grupo de los no citados, es el de una a cinco citas.

El tamaño de la muestra ha sido muy variado. El estudio en el que se analizó la mayor cantidad de muestra, se analizaron un total de 528 deportistas y en el que menos se analizaron 6 deportistas.

Respecto al nivel de la muestra, en muchos de los artículos no se ha podido comprobar el nivel de ésta, sólo en 40 de los artículos analizados. En la tabla 1, se observa que se ha encontrado que la gran mayoría estudian a deportistas profesionales. Los restantes el nivel de la muestra se reparten entre nivel amateur, y de varios niveles. De iniciación no se ha encontrado ningún artículo.

De los 48 artículos donde se ha podido comprobar el sexo de la muestra, se puede observar en la tabla 1 que la mayoría de ellos estudia deportistas de sexo masculino $(\mathrm{n}=36)$. La mujer como objeto de estudio en este deporte no resulta muy influyente, pues tan sólo 7 artículos toman a la mujer como muestra.

\section{Discusión}

Son muchos autores y de diferentes sitios del mundo los que aparecen en los artículos estudiados. Como ya se ha comentado anteriormente, es Travassos el autor que más investigaciones ha tenido respecto a este deporte en estas bases de datos. Por lo tanto, una de las instituciones que más artículos tiene es la de este autor, la Universidad Beira Interior, de Portugal.

Respecto a las instituciones, cabe decir que la mayoría 
de artículos proceden de universidades brasileñas $(\mathrm{n}=15)$, les siguen las universidades portuguesas, con 12 artículos. Estos datos son semejantes a los registrados por otros autores que analizaron esta modalidad deportiva (Moore, Bullough, Goldsmith y Edmondson, 2014). Estos resultados muestran que el deporte del fútbol sala tiene un gran auge en los países con habla portuguesa. Teniendo en cuenta los artículos de Portugal y Brasil, se aprecian 27 de las 57 instituciones que existen en los artículos analizados. El fútbol sala en Brasil es la segunda modalidad deportiva más popular de Brasil, solamente detrás del fútbol, y actualmente el deporte con mayor crecimiento en el mundo (Peres, Mesquita, Rombaldi y Cozzenssa, 2009).

La revista con mayor número de artículos publicados ha sido "Journal of Strength and Conditioning Research". Esta revista trata temas de condición física, por lo que se observa que las disciplinas predominantes son la Fisiología y la Teoría del Entrenamiento. En Este sentido, Moore et al. (2014), ya señalaron que la inmensa mayoría de los trabajos de investigación en los que se analiza el fútbol-sala, se estudian o bien aspectos relacioandos con la condición física, elementos fisiológicos, y en menor medida con las lesiones deportivas y aspectos psicológicos e indicadores de rendimiento técnicotácticos.

En cuanto al tipo de estudio utilizado en los artículos analizados, predomina el experimental por encima del descriptivo. En muchos estudios bibliométricos no se obtiene el mismo dato, donde predominan los estudios descriptivos (e.g. Balmaseda, 2014; Olmedilla, Ortega, Abenza y Boladeras, 2011; Peset, Ferrer-Sapena, Villamón, González, Toca-Herrera, y Aleixandre, 2013). Estas diferencias, pueden ser debidas a la propia temática principal de estudio, pues generalmente los estudios relacionados con la Fisiología y la Teoría del Entrenamiento, suelen ser de carácter experimental, frente a otros disciplinas de estudio, en las que suelen predominar otras metodologías.

Respecto al número de citas por artículo, el número de veces que es citado un libro o un artículo es un reflejo de su utilidad y del impacto de dicha publicación en la comunidad científica (Campanario, Cabos e Hidalgo, 1998; Ruiz-Pérez, Marcos-Cartagena, López-Cozar, 2014). Según estos autores, el número medio de citas por artículo español o latinoamericano es de 0,32 con autocitas y 0,08 sin autocitas. Siguiendo con estos autores, afirman que internacionalmente el número medio de citas por artículo con autocitas es de 3,60 y de1,86 sin autocitas. En este estudio se puede comprobar cómo la gran mayoría, 48,15\%, no han sido citados todavía, y el $32,09 \%$ de los artículos revisados han sido citados de 1 a 5 veces, por lo que este estudio y el de Campanario et al. (1998) coinciden en el número de citas que reciben los artículos.

Estudios como el de Marrugat, Vila, Pavesi y Sanz (1998) indican que trabajar con tamaños muestrales insuficientes es muy frecuente en este tipo de trabajos. Un alto porcentaje de los artículos analizados corresponde al grupo de menor tamaño (de 0 a 10). Tanto la temática principal de estudio, la Fisiología y la Teoría del Entrenamiento, como el tipo de estudio, estudio de carácter experimental, son los causante del bajo número de sujetos, pues este tipo de estudios, implica un coste muy superior tanto en recursos humanos como material, intrumentos, etc. El grupo que menos cantidad de artículos tiene es el de mayor muestral, más de 300.

Respecto al nivel de la muestra, se observa que existe un gran predominio en los estudios sobre jugadores profesionales, datos muy semejantes a los encontrados por Moore et al. (2014). En la actualidad la información que muestran diariamente artículos, periódicos y la propia televisión cómo la competición y el nivel profesional es el aspecto predominante sobre otros aspectos más formativos, y no sólo en nuestro país, sino también en países como Estados Unidos (Friederes y Palao, 2006).

En este estudio se muestra la inexistencia de artículos referidos a la iniciación deportiva dentro de la literatura, hecho muy relevante pues todos los grandes profesionales han pasado por un periodo formativo previo. Estudiar sobre el fútbol sala en niveles de iniciación puede ser muy interesante e importante para comprender mejor aspectos de los que ya se sabe a niveles profesionales. Existe la creencia de que el deporte de elite se ha convertido en un ámbito muy especializado (Torregosa, Sánchez y Cruz, 2004).

Por último, el estudio del género masculino está muy por encima del femenino, aspecto que sucede también en el estudio de Moore et al. (2014), así como en la inmensa mayoria de estudios bibliometricos en el ámbito deportivo, como por ejemplo en el estudio del deporte del Judo (Peset, Ferrer, Sapena, Villamón, Millán, Toca y Aleixandre 2013), Rugby (Villarejo, Palao y Ortega, 2010) o boxeo (Balmaceda, 2014). En este sentido se comprueba cada día cómo las noticias más vistas y buscadas son las relacionadas con el sexo masculino. Por cada noticia referida a mujeres hay 14,7- 15,6 noticias referidas a hombres (Friederes y Palao, 2006). En este sentido, los medios de comunicación demuestran que las mujeres deportistas no alcanzar el 2\% del tiempo de voz (López, 2011; Sainz de Baranda, 2014).

\section{Conclusiones}

\section{Como conclusión se puede decir que}

- Los autores e instituciones con mayor número de artículos son de países con habla portuguesa (Brasil y Portugal).

- La gran mayoría de los artículos toman como muestra al sexo masculino y a niveles profesionales.

- La mayoría de artículos todavía no han sido citados, pero existen 4 artículos con más de 20 citas. 
- Los años en los que más cantidad de artículos se ha publicado han sido 2012 y 2013.

- La revista con más artículos publicados es "Journal of Strength and Conditioning Research",

- El tema de mayor interés en el fútbol sala es la condi- ción física, pues las disciplinas más estudiadas corresponden con esto (Fisiología y Teoría del Entrenamiento).

- Respecto al tipo de estudio predomina el experimental por encima del descriptivo.

\section{Referencias Bibliográficas}

1. Arbinaga, F., Aragón, J.D., y Tejedor, R. (2010). Análisis bibliométrico de la Revista de Psicología del Deporte (1992-2009). Revista de Psicología del Deporte, 19(2), 231-245

2. Balmaseda, M. (2014). Análisis de las acciones técnico-tácticas del boxeo de rendimiento. Tesis Doctoral. Pais Vasco: Servicio Editorial de la Universidad del País Vasco.

3. Campanario, J., Cabos, W., y Hidalgo, M. (1998). El impacto de la producción científica de la Universidad de Alcalá de Henares. Revista española de documentación científica, 21(4), 402-415.

4. Carrizo, G. (2000). La información en ciencias sociales. Gijón: Trea, S.L.

5. Devís, J., Antolín, L., Villamón M., Moreno, A., y Valenciano, J. (2003). Las revistas científicas-técnicas espańolas de las ciencias de la Actividad Física y el Deporte: inventario y análisis de la calidad de contenido y difusión. Revista española de Documentación Científica, 26(2), 177-190.

6. Devís, J., Valenciano, J., Villamón, M., y Pérez, V. (2010). Disciplinas y temas de estudio en las ciencias de la actividad física y el deporte. Revista Internacional de Medicina de Ciencias de la Actividad Física y del Deporte.10(37), 150-166.

7. Devís, J., Villamón, M., Antolín, L., Valenciano, J., y Moreno, A. (2004). Las revistas científico-técnicas españolas de ciencias de la actividad física y el deporte: adecuación a las normas ISO y grado de normalización. Ciência da Informação, 33(1), 38-47.

8. Estrada, Y. (2013). Estudio exploratorio sobre el estado del arte en la rehabilitación deportiva en Colombia. Cuerpo, Cultura y Movimiento, 3(5), 79-110.

9. Fernández, A., y Bueno, A. (1998). Síntesis de estudios bibliométricos españoles en educación. Una dimensión evaluativa. Revista española de Documentación Científica. 21(3), 269-285.

10. Fernández, S., y Alvero, R. (2006). La producción científica en cineantropometría: datos de referencia de composición corporal y somatotipo. Archivos de Medicina del Deporte, 23(1), 17-35.

11. Friederes, J., y Palao J.M. (2006). Análisis de las noticias deportivas de dos periódicos digitales de Espańa y Estados Unidos: ipromoción de la actividad física y el deporte? Apunts: Educación Física y Deportes, 85(1), 7-14.

12. González, M. (2004). El reflejo del deporte en los medios de comunicación en España. Revista Española de Sociología,4, 271-280.

13. Hernández, R., Fernández, C., y Baptista, L. (2000). Metodología de la investigación (2a Edición ampliada). México: MC Graw Hill.

14. López, A. (2011). Deporte y mujeres en los medios de comunicación. Sugerencias y recomendaciones. Universidad de Castilla La Mancha: underbau.

15. Marín, E., Ortín, F. J., Garcés de Los Fayos, E. J., y Tutte, V. (2013). Análisis bibliométrico de burnout y optimismo en deporte. Sportk, 2(1), 77-87.

16. Marrugat, J., Vila, J., Pavesi, M., y Sanz, F. (1998). Estimación del tamańo de la muestra en la investigación clínica y epidemiológica. Medicina Clinica, 111(7), 267-276.

17. Martínez, A., Moya, F.J., y Garcés de los Fayos, E.J. (2013). Inteligencia emocional y deporte: Situación actual del estado de la investigación. Cuadernos de psicología del deporte, 13(1), 105-112.

18. Martínez, J.A, Ruiz, J., y Navarro, R. (2002). Análisis documental y bibliométrico sobre el arbitraje en el fútbol en la base de datos sportd- iscus. 1830-2002. Revistas" Jornadas canarias de traumatología y cirugía ortopédica para especialistas y post-graduados», 16, 199-200.

19. Moore, R., Bullough, S., Goldsmith, S., \& Edmondson, L. (2014). A systematic review of futsal literature. American Journal of Sports Science and Medicine, 2(3), 108-116.

20. Olmedilla, A., Ortega, E., Abenza, L., y Boladeras, A. (2011). Lesiones deportivas y psicología: una revisión (2000-2009). Cuadernos de Psicología del Deporte, 11(1). 45-57.

21. Olmedilla, A., Ortega, E., González, J., y Villarejo, D. (2013). Análisis de los proyectos de investigación de financiación pública en Psicología del Deporte. Anales de psicología, 29(3), 714-723.

22. Ortega, E., Valdivia, P., Villarejo, D., y Olmedilla, A. (2014). Análisis de los proyectos de investigación concedidos por el Consejo Superior de Deportes (2006-2012), desde una perspectiva de género. Revista de Psicología del Deporte, 23(1), 95-100.

23. Peres, A., Mesquita, L., Rombaldi, A., y Cozzenssa, M. (2009). Eficiencia de 4 semanas de pre-temporada en jugadores de futsal en la preparación para el Campeonato Sudamericano de Clubes. Lecturas: Educación fisica y deportes, 136, 1-8.

24. Pérez, V. (2007). Análisis de las contribuciones a los congresos del área de Didáctica de la Expresión Corporal (1990-2000). Apunt: Educación física y deporte 87(1), 27-34.

25. Peset, F., Ferrer, A., Villamón, M., Millán, L., Toca, J., y Aleixandre, R. (2013). Scientific literature analysis of Judo in Web of Science. E-prints in library \& information science, 9(2), 81-91.

26. Rodríguez, M., Rodríguez, C., Torres, A., y Urrego, C. (2011). Dinámica de las investigaciones sobre el uso de la bio-retroinformación en el rendimiento deportivo. Cuadernos hispanoamericanos de psicología, 11(2), 79-92.

27. Ruiz-Pérez, R., Marcos-Cartagena, D., López-Cózar, E. (2014). La autoría científica en las áreas de ciencia y tecnología. Políticas internacionales y prácticas editoriales en las revistas científicas españolas. Revista Española de Documentación Científica, 37 (2), e049, doi:10.3989/redc

28. Sainz de Baranda, C. (2014). Las mujeres en la prensa deportiva: dos perfiles. Cuadernos de Psicología del Deporte, 14(1), 91-102.

29. Torrebadella, X. (2012). L'aportació dels metges de Lleida a l'educació física catalana del segle XIX. Temps d'Educació, 43, 109-130.

30. Torregrosa, M., Sánchez, X., y Cruz, J. (2004). El papel del psicólogo del deporte en el asesoramiento académico - vocacional del deportista de elite. Revista de psicología del deporte, 13(2), 215-228.

31. Villamón, M., Devís, J., y Valenciano, J. (2005). Análisis de la visibilidad de las revistas científico-técnicas espańolas de ciencias de la actividad física y el deporte Revista de Psicología del Deporte, 14(2), 253, 267.

32. Villamón, M., Devís, J., Valencia, A., y Valenciano J. (2007). Características y difusión de las revistas científico-técnicas españolas de ciencias de la actividad física y el deporte. El profesional de la información revista, 16(6), 605-615.

33. Villarejo, D., Palao, J., y Ortega, E. (2010). La producción científica en rugby union entre 1998-2007. E-Balonmano: Revista de Ciencias del Deporte, 6(3), 155.161.

34. Zulueta, M., Cabrero, A., y Bordons, M. (1999). Identificación y estudio de Grupos de Investigación a través de indicadores bibliométricos. Revista española de Documentación Cientifica, 22(3), 333-347. 